\title{
Non-Line of Sight LoRa -Based Localization using RSSI-Kalman-Filter and Trilateration
}

\author{
Thirafi Wian Anugrah ${ }^{1 *}$, Andrian Rakhmatsyah ${ }^{1}$, Aulia Arif Wardana ${ }^{1}$ \\ ${ }^{1}$ School of Computing, Telkom University \\ Bandung, Indonesia \\ *thirafiwian@student.telkomuniversity.ac.id
}

\begin{abstract}
This research builds a Localization System using Long Range (LoRa) Network. This research also analyzes the performance of the positioning method in the Low-Power Wide-Area Network (LPWAN). LPWAN technology is radio-based communication that low power usage and wide area coverage. The method that analyzes in this research is the combination of the Received Signal Strength Indicator (RSSI) with the Trilateration Method. This research also filtered the RSSI value using the Kalman filter method for smoothing data. The localization system traditionally based on Global Positioning System (GPS) device. However, GPS technology not working well in Non-lineof-sight (NLOS) like an indoor location or mountain area. The other way to implement the localization system is by using LoRa technology. This technology used radio frequency to communicate with each other node. The radio frequency has a measurement value in the form of signal strength. These parameters, when combined with the trilateration method, can be used as a localization system. After implementation and testing, the system can work well compared with the GPS system for localization. RMSE is used to calculate error position on these methods, the result from three methods used, the value from RSSI with Kalman filter have a close result to actual position, then value GPS follows with close result from Kalman filter, and the last one is RSSI without Kalman filter.
\end{abstract}

Keywords: Localization, Object Tracking, LoRa, GPS, Trilateration, RSSI, Kalman Filter

\section{INTRODUCTION}

$\mathbf{T}$ he usage of LPWAN in recent years is rising for communication across the sensor node in Wireless Sensor Network (WSN) technology [1] Low-Power Wide-Area Network (LPWAN) is radio frequency technology that can transmit long-range signals for wide area coverage using low power resources. This feature is very powerful for indoor or outdoor communication protocol with a wide area and many obstacles [2]. One of the technologies that implement the LPWAN mechanism is LoRa. This technology is very cheap for LPWAN technology compared to Sigfox or NB-IoT [3], [4]. LoRa used unlicensed radio frequency and each country has the regulation about the LoRa radio frequency.

The LoRa technology can reach up to $5 \mathrm{KM}$ in an urban area and $15 \mathrm{~km}$ in rural areas [5], [6]. LoRa network topology can be applied in two modes, there is star and mesh topology. Each topology has a gateway that used to get all data from all nodes that connect in LoRa Network. The gateway is also useful as a packet forwarder from non-IP based network to an IP based network. This research will used mesh topology for communication between nodes. A single node (end node) when transmitting data to gateway need to communicate with each other node. Each other node with the closest distance will forward data from the end node to the gateway. The 
other node that closest distance and help end node to forward data called by Base Station (BS) [7]. The LoRa network have some configuration in physical layer, there are Carrier Frequency (CF) for setting the radio frequency from LoRa, Spreading Factor (SF) for determining the number of chirps used to represent a symbol, Bandwidth (BW) for representing the amount of frequency when transmitting, Coding Rate (CR) for handle packet error rate [8], [9].

One feature in radio-based communication is can be used as a localization system. The localization system usually using GPS to get the latitude and longitude from objects to mark its position on earth [10], [11]. GPS has some drawbacks when detecting the location in the NLOS environment. First, The GPS not working well in indoor location because the satellite signal will block by building material like brick, metal, stone or wood [12], [13]. Second, The GPS considers the mountains or hilly area to be a noise because the contour of the area is bumpy so the GPS is difficult to get signal from satellite [14]. Based on the problem, this research uses other technology to implement a localization system that supports for NLOS environment.

This research will use LoRa to build a localization system. LoRa is based on radio frequency, the radio signal from LoRa has a Received Signal Strength Indicator (RSSI) as signal strength parameter [15], [16]. The parameter can be used to determine the position of the object when it enters the LoRa network. The advantage used of LoRa for the localization system is a wide range of area coverage and low power usage. The other advantage is LoRa is cheap and easy for use because of no requiring additional processing power [17].

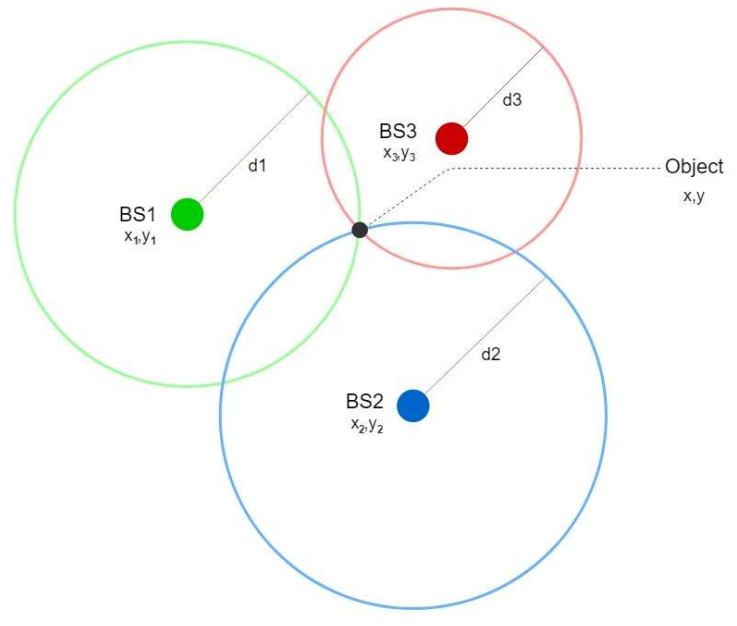

Fig. 1. Determine position from object using RSSI (N. A. Azmi et al, 2018)

The popular method for localization system based on radio frequency is RSSI, but this method needs an algorithm to process RSSI data into a location of objects [18]. Trilateration can process RSSI values that have been converted into distances to be processed into coordinates of objects. This algorithm needs a three-point coordinate of BS from LoRa [19]. Each BS from LoRa has a radius, so it can be described as a circle. Trilateration is calculated as the intersection point of the three circles from LoRa BS to determine the $(x, y)$ coordinate from the object. The determination of the position of an object using three BS of LoRa can be seen in Fig 1. 


\section{LITERATURE REVIEW}

The research proposed a new RSSI-based localization method using LoRa Network. The research proposed a localization method for the noisy outdoor environment. The research proposed a new algorithm for improving the localization method in noisy outdoor environments.

The other research about the LoRa-based localization method using RSSI and trilateration. The research experiment in an area without obstacle and other inference. The research also comparing the localization method using GPS [20]. The last research about localization in the LoRa network using the RSSI method and combine with some algorithm. The research comparing algorithms that combining with RSSI. The algorithm that compared is trilateration, min-max, and maximum likelihood. The experiment is in indoor and outdoor environments [21].

TABLE I

RESEARCH FOCUS AND WEAKNESS FROM RELATED RESEARCH

\begin{tabular}{|c|c|c|}
\hline Authors & Research focus & Weakness \\
\hline $\begin{array}{l}\text { Marina Md. Din, } \\
\text { Norziana Jamil } \\
\text { and Nik Fariz } \\
\text { Nik Ahmad Aziz } \\
\text { [19] }\end{array}$ & $\begin{array}{l}\text { Centroid Localization Algorithm that } \\
\text { can manipulate noise signals from raw } \\
\text { Received Signal Strength Indicator } \\
\text { (RSSI). }\end{array}$ & $\begin{array}{l}\text { Using mobile device communication and not } \\
\text { using an embedded system that makes noise } \\
\text { manipulation already performed on the } \\
\text { device, while the beacon node still uses an } \\
\text { access point that has noise correction that } \\
\text { resembles a mobile device }\end{array}$ \\
\hline $\begin{array}{l}\text { W. Bulten, A. C. } \\
\text { V et al [22] }\end{array}$ & $\begin{array}{l}\text { Using enhanced Kalman filter to } \\
\text { reduces spikes data. }\end{array}$ & $\begin{array}{l}\text { Tested on indoor area and number of noises } \\
\text { created from one source, made the data } \\
\text { already fixed on several point tested. }\end{array}$ \\
\hline
\end{tabular}

From table 1 can concluded on the previous research that focus on Trilateration and using Kalman filter, few of researches didn't merge the result from Trilateration and Kalman filter which have an accuracy based on some method, and the technology used on previous research didn't use LoRa as main device to test, previous research also tested on indoor area which have few noises factor different from this research that tested on outdoor area that have a large of number noises factor such as trees, building, and much more.

On this research based on the other research, there is no filter for the RSSI noise during the process with the trilateration algorithm as a localization method. This research conduct to filter RSSI using Kalman filter before processing with a trilateration algorithm, and this research will show high accuracy localization by merging these methods on NLOS area using LoRa.

This study uses the calculation of Root Mean Square Error (RMSE) to validate output from Kalman filter and Trilateration method, validation is done to ensure that the results of this mixed method it is produce high accuracy and low error between predictive positions to actual positions rather than GPS position localization on NLOS environment [23].

\section{RESEARCH METHOD}

This research begins with the introduction section that explains about identify the problem from the traditional localization system in a wide area and NLOS environment. The introduction section also explains the alternative system that can implement the localization systems. Then the literature review section explained and comparing this research with research that has been done. After identifying the problem, in the research methods section, this research study more detailed about the method used in the localization system using radio frequency that 
suitable for LoRa technology. In the next step, in the result and discussion section, this research implements the LoRa technology with the localization method based on radio frequency. The section also explains the testing scenario and testing result for performance evaluation of implementation localization method in LoRa network.

\section{A. Localization Techniques}

This research will use the RSSI method to determine the position of the object. Usually, the RSSI value of the radio signal in LoRa has a noise that must be filtered. The noise is caused by obstacles, consequently the LoRa signal to be disturbed. In the NLOS environment, the obstacle will be very numerous and very disturbing signals from LoRa. This research will use the Kalman filter method to filter the noise from RSSI value in LoRa [24].

To address the noise problem a Kalman Filter is applied to filter signal strength measurements. The Kalman filter works by estimating the ideal RSSI value on a radio signal by using the previous RSSI value model, the general form of the transition model is like equation 1[22].

$$
x_{t}=A_{t} x_{t_{-1}}+B_{t} u_{t}+\epsilon_{t}
$$

where $x_{t}$ is RRSI value that combination from $x_{t_{-1}}$ in matrix transformation $A . u$ is input control and $\epsilon$ is noise value. In this research, device will stop in 10 second in particular moment, in the moment the $u$ value will ignore and set $A$ as a matrix identity, finally a simple model is formed in the equation 2 .

$$
x_{t}=A_{t} x_{t_{-1}}+B_{t} u_{t}+\epsilon_{t} \approx x_{t_{-1}}+\epsilon_{t}
$$

The equation 2 is applied to estimate how much needs to be normalized from noise at the RSSI value.

Each BS will spread the radio signal to scan the coverage range. When the object (node with radiofrequency) enter into coverage range from BS, the object will convert RSSI from each BS to distance value in a meter. After filter RSSI data with the Kalman filter, then the RSSI value will convert to distance. The equation in equation 3 explains how to convert from RSSI to distance.

$$
d=10^{\frac{(A-R S S I)}{(10 * n)}}
$$

Where $d$ is distance between base station and end node in meter, then $A$ is the signal strength which is received from reference nodes (end node) at the distance in $\mathrm{dBm}$, RSSI is RSSI value in $\mathrm{dBm}$, and the last $n$ is constant and the value depends on the environmental factor, this research used default value of 2 .

After each distance from BS is calculated by the end node, the location of the object can determine, but just by distance. Figure 1 is an example of how the RSSI can determine the location of the object by convert RSSI to distance.

The next step is to calculate the distance using a trilateration algorithm to detail the position from the object. This algorithm is commonly used to determine the position based on the distance between transmitter and receiver. This algorithm is very suitable for the RSSI method because we can calculate the distance between 
the transmitter (base station) and receiver (end node) using RSSI. This algorithm is a geometry-based algorithm. Equation 4 explains about trilateration algorithm for calculate position using RSSI.

$$
\left(x-x_{i}\right)^{2}+\left(y-y_{i}\right)^{2}=d_{i}^{2}
$$

Where $d i$ is distance between base station and end node in meter and $i$ is ID from base station. Then $x i$ is base station $X$ axis coordinate and $\mathrm{i}$ is ID from base station. After that $y i$ is base station $Y$ axis coordinate and $i$ is ID from base station. And finally, $x$ and $y$ are actual coordinate from object (end node) which will be computed.

This research will use three base stations to determine the position of the object. The iteration from equation 4 will repeat three times for three different base stations. Based on Fig 1 and equation 4, this research has three equations for three circles. The equation can see in equation 5, 6, 7 .

$$
\begin{aligned}
& \left(x-x_{1}\right)^{2}+\left(y-y_{1}\right)^{2}=d_{1}^{2} \\
& \left(x-x_{2}\right)^{2}+\left(y-y_{2}\right)^{2}=d_{2}^{2} \\
& \left(x-x_{3}\right)^{2}+\left(y-y_{3}\right)^{2}=d_{3}^{2}
\end{aligned}
$$

The three equation in equation 5, 6, 7 can be expanded and becomes equation $8,9,10$.

$$
\begin{gathered}
x^{2}-2 x_{1} x+x_{1}^{2}+y^{2}-2 y_{1} y+y_{1}^{2}=d_{1}^{2} \\
x^{2}-2 x_{2} x+x_{2}^{2}+y^{2}-2 y_{2} y+y_{2}^{2}=d_{2}^{2} \\
x^{2}-2 x_{3} x+x_{3}^{2}+y^{2}-2 y_{3} y+y_{3}^{2}=d_{3}^{2}
\end{gathered}
$$

After that, subtract equation in equation 9 from the equation in equation 8, the result becomes equation 11 . Likewise, subtract equation in equation 10 from the equation in equation 9, the result becomes equation 12 .

$$
\begin{aligned}
& \left(-2 x_{1}+2 x_{2}\right) x+\left(-2 y_{1}+2 y_{2}\right) y=d_{1}^{2}-d_{2}^{2}-x_{1}^{2}+x_{2}^{2}-y_{1}^{2}+y_{2}^{2} \\
& \left(-2 x_{2}+2 x_{3}\right) x+\left(-2 y_{2}+2 y_{3}\right) y=d_{2}^{2}-d_{3}^{2}-x_{2}^{2}+x_{3}^{2}-y_{2}^{2}+y_{3}^{2}
\end{aligned}
$$

The equation in equation 11 and 12 is too long. The two-equation will rewrite using $G, H, I, J, K$, and $L$ as a symbol to simplify the equation. The result from the simplifying equation become equation 13 and 14 .

$$
G x+H y=I
$$




$$
J x+K y=L
$$

The solution from determine the $X$ and $Y$ position from the object become equation 15 and 16.

$$
\begin{aligned}
& x=\frac{I K-L H}{K G-H J} \\
& y=\frac{I J-G L}{H J-G K}
\end{aligned}
$$

The localization technique method based on RSSI and trilateration used equation 15 and 16 to compute the position from an object in LoRa Network for the localization system.

\section{RESULTS AND DISCUSSION}

\section{A. System testing environment.}

This research used Dragino LoRa Arduino shield for BS to detect an object. The object is equipped with a LoRa Sx1276 module with a $915 \mathrm{Mhz}$ frequency configuration and uses ESP32 microcontroller. The gateway from each node using the Dragino LoRa raspberry hat. The gateway also used as the main BS and the task is getting all data from each other BS to process the data. The antenna from LoRa in each node (BS, Gateway, End Node) used $3 \mathrm{dBi}$. The power resource for each node and end node (except gateway) used $9 \mathrm{~V}$ battery, the gateway used $12 \mathrm{~V}$ adaptor power resource.

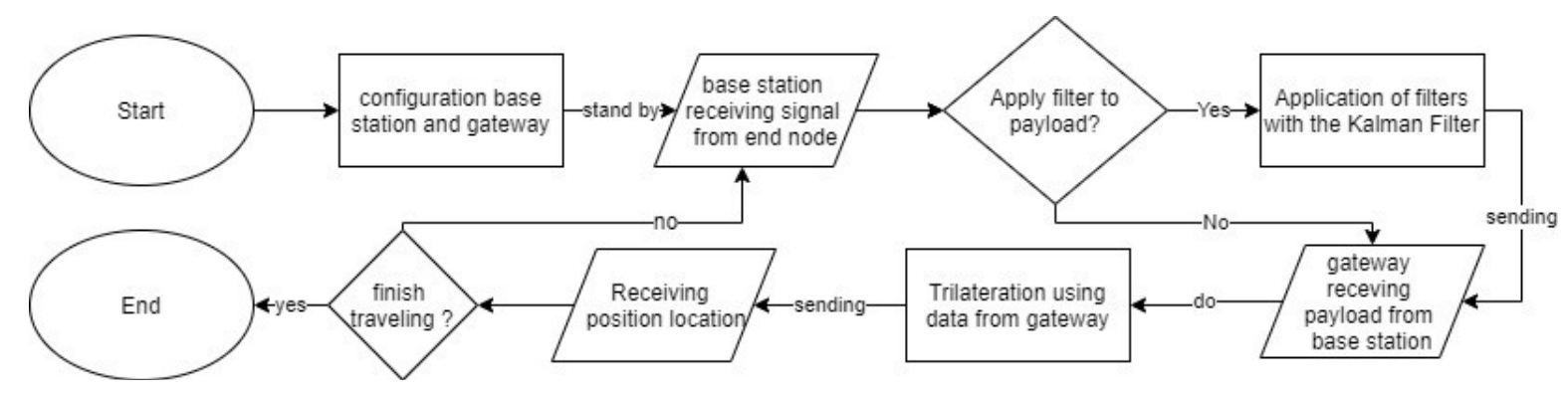

Fig. 2. Experiment setup.

The setup for this experiment based on the flow at fig 2 explained how base station and gateway were setup and calibrate to tested on the field as fig 3 showed. Then during the trip, the base station receives a signal from the end node which will then be forwarded to the gateway. Because some of the data have a bad condition because of the noises, then applying Kalman Filter is needed to normalize the data. After being filtered, the data will become a parameter to Trilateration to predict the exact location of end node position.

The Spreading factor (SF) configuration from LoRa used SF 9 and the frequency is $915 \mathrm{Mhz}$. This configuration was chosen because it has a good packet delivery ratio for distances of 240 meters, has a low packet error rate for distances of 240 meters, and has an RSSI mean value not more than $-95.16 \mathrm{dBm}$ for distances of 240 meters. The 240 meters distance represented the maximum distance between each base station in our trial area. The GPS used in this research for comparison data is getting from mobile phone GPS data. The specification is Samsung type A10 with operating system Android version 9 and GPS version 10.34.3. 


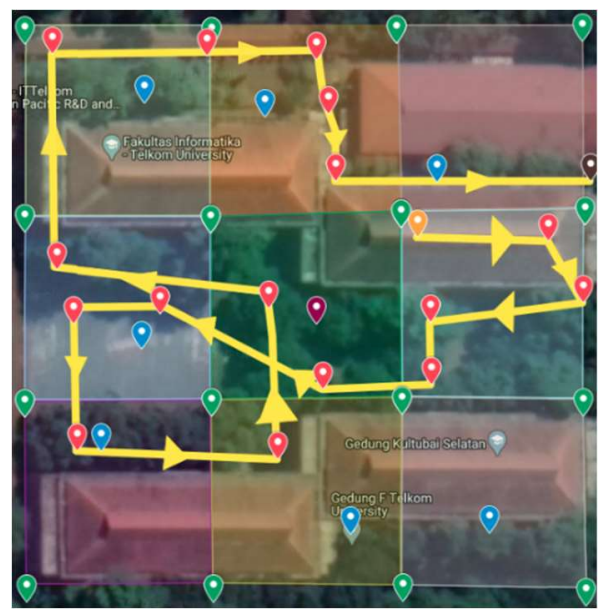

Fig. 3. Testing area for NLOS environment.

\section{B. Experiment environment and Location Overview}

The testing area is located at Telkom University, Bandung, Indonesia (-6.976532, 107.630788). The arrangement of all BS will be arranged as shown in Fig 3. The BS is denoted by a blue point and the gateway is denoted by magenta point in fig 3 . After placing all base station and gateway, fig 3 can map the grid from location coverage. The grid that builds by green point is the coverage area from this localization system. The distance between each green point in fig 3 is 30 meters. This means one BS that doted by blue point representing four green points as coverage area. As can be seen on the maps in Fig 3, there are various kinds of obstacles in the localization system. There are several buildings, trees and other objects that can interfere with signals from the LoRa network. That area is enough to represent the NLOS environment to test the system in this study because from the fig 3 , the environment of test area has multiple building and trees that can caused NLOS and noises to the signal.

\section{Testing Result}

The test measured in this study is accuracy for distance determination in the localization method. The accuracy of the method proposed for localization will be compared with GPS. The first step for the testing mechanism is to make an object travel plan using Google My Maps. The object travel plan will form a path for testing the movement of the object. The path from an object travel plan can see in fig 3 .

In Fig 3, the orange point is the start point and the brown point is the finish point from an object travel plan. The yellow line is the object moving path from start point to finish point. The length of the object's movement path (yellow line) is 397 meters. The object is equipped with LoRa and GPS, then the object will travel from orange point to brown point based on the direction of the object's movement path (yellow line). Along the path of object movement, there is some stopping point that dotted by a red point. The stop point is used to get stable GPS (latitude and longitude) and LoRa (RSSI) data. The object will stop at a stopping point with approximately 5-10 seconds. After getting all data from GPS and LoRa, then all the data will be converted into a path. All produced paths will issue a distance value. The distance value from all produced path can see in Table 1. 
TABLE II

Path distance DATA FRom ACtUAL, GPS, AND LoRA DATA

\begin{tabular}{|c|c|c|c|c|}
\hline $\begin{array}{l}\text { Actual } \\
\text { (M) }\end{array}$ & Distance & $\begin{array}{l}\text { LoRa Kalman } \\
\text { Filter (M) }\end{array}$ & $\begin{array}{l}\text { LoRa No Filter } \\
\text { (M) }\end{array}$ & GPS (M) \\
\hline $397 \mathrm{M}$ & & $377 \mathrm{M}$ & $306 \mathrm{M}$ & $289 \mathrm{M}$ \\
\hline
\end{tabular}

The distance from Table 2 shows that the distance from the path created with LoRa Kalman filter close to the actual distance. This means LoRa with Kalman filter can make a better object path for tracking in the localization system. The visualization from a path created with the Kalman filter can see in figure 3 . The path from an object moving in figure 3 represented with red line. The path is created using LoRa RSSI that filtered with Kalman filter and processing using the trilateration method. The blue point in figure 3 is the position from $\mathrm{BS}$, the BS position is the same with a blue point in fig 3. Based on figure 3, RSSI that filtered with Kalman filter and process using the trilateration method can know positioning from the object and can make tracking path from the object. This means LoRa is suitable for the localization system because it knows the tracking path from the object.
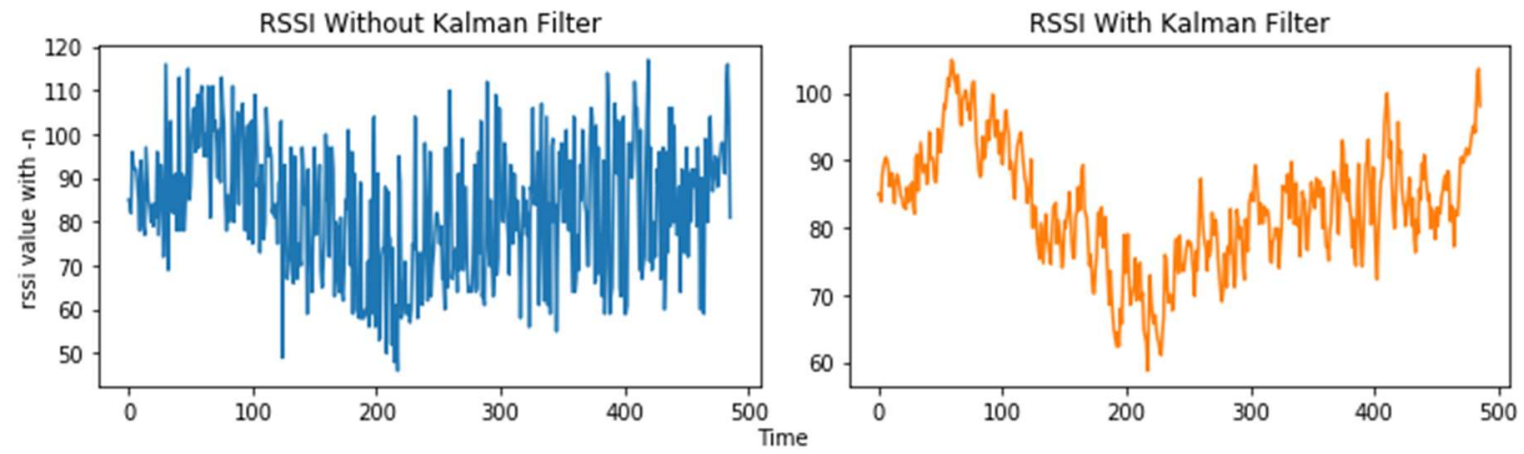

Fig. 4 Kalman Filter implementation

The graph in the fig 4 is the data entries from the end node, then received to the nodes, then the data is collected to the gateway, after that the RSSI graph data without the Kalman filter has messy value because of the environment that caused noises as showed at fig 3, while RSSI value using Kalman filter more tidy and normalized data, it is used Kalman filter to reduce the possibility of data that has multiple data or unstable RSSI values in the payload data of a particular node.

The other testing measures the error value from latitude and longitude data from GPS, RSSI-LoRa without Kalman filter, and RSSI-LoRa with Kalman filter compared with latitude and longitude data from Google My Maps application. The error measurement will measure with equation 17.

$$
\sum E r r=\frac{r v-m v}{r v} * 100 \%
$$

Where Err is error value from latitude or longitude data in percentage (\%). $r v$ is the reference value from latitude or longitude data. This research used Google My Maps as reference data. $m v$ is the measurement value from latitude or longitude data. This research used GPS and $X, Y$ data from LoRa with kalman filter and without kalman filter that already convert to latitude and longitude data.

Before comparing latitude and longitude data, the X,Y position from the object that process with trilateration based on RSSI-LoRa without Kalman filter data and RSSI-LoRa with Kalman filter data must be converted to latitude and longitude format. The equation for convert X,Y position to latitude and longitude GPS data using the equation in equation 18-20. 


$$
\begin{gathered}
X=(v+h) \cos \phi \cos \\
Y=(v+h) \cos \lambda \sin \\
Z=\left(v\left(1-e^{2}\right)+h\right) \sin
\end{gathered}
$$

where $(v+h) \cos$ is the radius from the earth, $\phi \cos$ is latitude value and $\lambda \sin$ is longitude value, $Z$ value just to be valued from earth pole, and $e$ is value of previous distance of $\mathrm{X}$ and $\mathrm{Y}$.

TABLE II

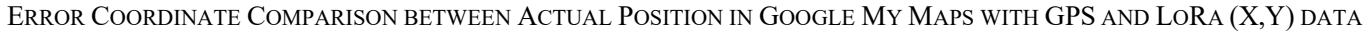

\begin{tabular}{cccccc}
\hline \multicolumn{2}{c}{ Error on moving GPS } & \multicolumn{2}{c}{ Error on RSSI without Kalman Filter } & \multicolumn{2}{c}{$\begin{array}{c}\text { Error on RSSI with Kalman } \\
\text { Filter }\end{array}$} \\
\hline $\begin{array}{c}\text { Error on } \\
\text { Latitude (\%) }\end{array}$ & $\begin{array}{c}\text { Error on } \\
\text { Longitude (\%) }\end{array}$ & $\begin{array}{c}\text { Error on Latitude } \\
(\%)\end{array}$ & $\begin{array}{c}\text { Error on } \\
\text { Longitude (\%) }\end{array}$ & $\begin{array}{c}\text { Error on } \\
\text { Latitude (\%) }\end{array}$ & $\begin{array}{c}\text { Error on } \\
\text { Longitude (\%) }\end{array}$ \\
\hline 0.00000287 & 0.00000046 & 0.00000287 & 0.00000084 & 0.00000143 & 0.00000009 \\
0.00000143 & 0.00000065 & 0.00000717 & 0.00000074 & 0.00000143 & 0.00000019 \\
0.00000287 & 0.00000056 & 0.00000143 & 0.00000158 & 0 & 0.00000037 \\
0.00000717 & 0.00000056 & 0.00000287 & 0.00000046 & 0.00000143 & 0 \\
0.00000287 & 0.00000028 & 0.00000143 & 0.00000056 & 0.0000043 & 0.00000019 \\
0.00000143 & 0.00000037 & 0.00000287 & 0.00000046 & 0 & 0.00000121 \\
0.00000287 & 0.00000056 & 0.00001433 & 0.00000037 & 0.00000143 & 0.00000046 \\
0.00001147 & 0.00000037 & 0.00001003 & 0.00000037 & 0 & 0 \\
0.00000143 & 0.00000056 & 0.00000573 & 0.00000046 & 0.0000086 & 0.00000037 \\
0.00000573 & 0 & 0.00000717 & 0.00000065 & 0.00000287 & 0.00000037 \\
0.0000043 & 0.10000028 & 0.00000717 & 0.00000028 & 0 & 0.00000037 \\
0.00000573 & 0.00000065 & 0.0000043 & 0.00000065 & 0.00000287 & 0.00000084 \\
0.00001003 & 0.00000056 & 0.0000086 & 0.00000093 & 0.00000143 & 0.00000019 \\
0.00000717 & 0.00000046 & 0.00000287 & 0.00000102 & 0.00000573 & 0.00000037 \\
0.00000143 & 0.00000046 & 0.00000287 & 0.0000013 & 0 & 0 \\
0.00000717 & 0.00000065 & 0.00000287 & 0.00000037 & 0.0000043 & 0.00000056 \\
0.00000287 & 0 & 0 & 0.00000037 & 0.00000287 & 0.00000037 \\
\hline
\end{tabular}

Table 2 shows the results of the error value using equation 17, by using equation 17, the latitude and longitude error position were calculated based on the real latitude and longitude. From the result of table 2 is concluded that the difference in position points between GPS, RSSI and Kalman filter, and without the Kalman filter to the actual position, the value of each method has a significant result due to the output generated by Trilateration and the environment from the test area.

The result from latitude and longitude data from every stop point in fig 3 is the GPS data has an average latitude error value of $0.00046376 \%$ and an average longitude error value of $0.588279 \%$. The RSSI without Kalman filter has an average latitude error value of $0.00049753 \%$ and an average longitude error value of $0.00006712 \%$. The RSSI with Kalman filter has an average latitude error value of $0.00022758 \%$ and an average longitude error value of $0.000035 \%$. Based on these data it can be concluded that the latitude and longitude errors in RSSI using Kalman filters are less than GPS and RSSI without Kalman filters, and as the result of the trilateration can be seen at fig 5 where the green dots are predicted position based on the result from trilateration and Kalman Filter. 


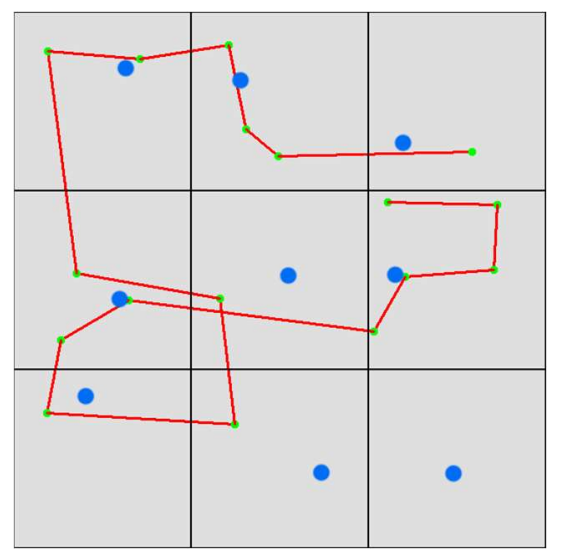

Fig. 5. Path predicting using RSSI data with kalman filter.

Then to get the error value from the actual position from predicted position that the date obtained from RSSI and mobile GPS for evaluating the result prediction result from actual position, equation 21 is used to test whether the value of the resulting distance approaches the actual position.

$$
R M S E=\frac{1}{T} \sum_{t=1}^{T} \sqrt{(\hat{x}(t)-x(t))^{2}+(\hat{y}(t)-y(t))^{2}}
$$

where $x(t)$ and $y(t)$ are pinpoint position, while $\hat{x}(t)$ dan $\hat{y}(t)$ predicted position from LoRa dan GPS, while T is data gathered from gateway, and the result showed at fig 6.

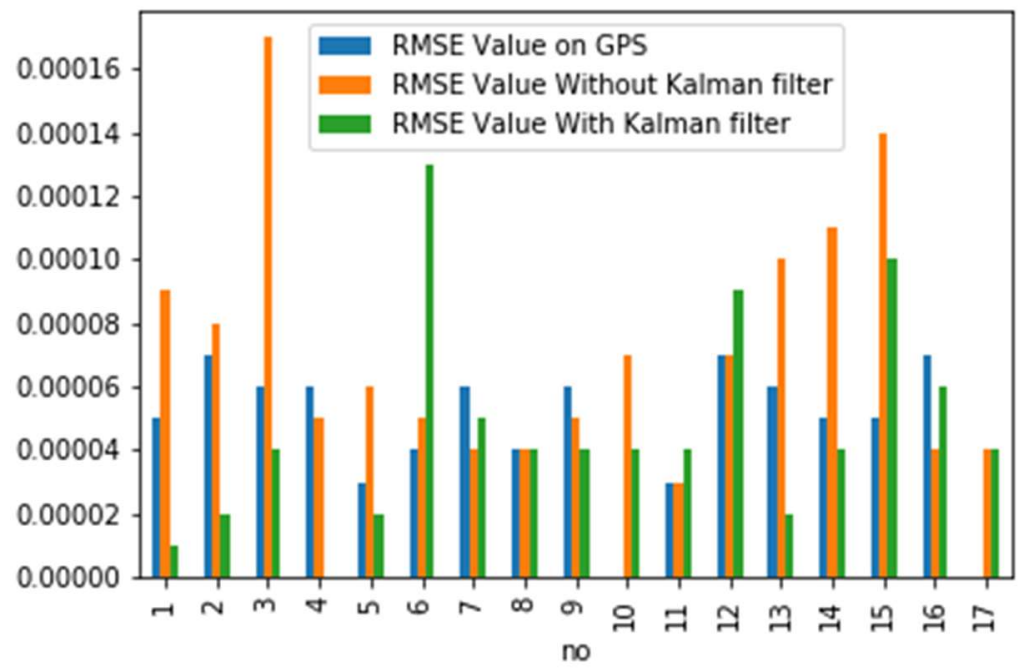

Fig. 6. RMSE value comparation between GPS data, RSSI without Kalman filter, and RSSI with Kalman filter .

Where the $\mathrm{y}$ axis is the number values of error and the $\mathrm{x}$ axis is each actual position from tested location, from fig 6 concluded, that each trilateration of the test location on the fig 3 with the actual GPS points have different accuracy between the mobile GPS, RSSI with the Kalman filter, and RSSI without the Kalman filter, this difference occurs because each position of the trial has different noises on NLOS on each of the LoRa and GPS devices, this causes interference with RSSI and GPS data on mobile phones. The graph on fig 6 showed that RSSI data using Kalman filter have a less RMSE Value other than GPS and Non Kalman Filter. 


\section{CONCLUSION}

This research succeeded in applying the localization system for NLOS environment using combination of the filtered using Kalman Filter, RSSI method and trilateration method in LoRa Network on NLOS area. This research also proof that LoRa is suitable for localization system in NLOS environment, with the condition as showed at fig 3 that all areas covered by LoRa network signal. The RSSI value that filter using Kalman filter method can improve the path for an object. The value from RMSE method also showed that RSSI using Kalman filter have a better result than other methods which have the minimum error from the actual positioning as shown at fig 6 . And the distance from the path created by RSSI filtered using the Kalman filter to be close to the actual distance with error value is $5.0 \%$. The distance path that created using non-filtered RSSI have an error value $22.9 \%$ when compared with actual distance. The worst results are shown by the distance from the path made by GPS with error value is $27.2 \%$. Which the result showed that this research have higher accuracy than previous research with same method but without merging trilateration and Kalman filter.

\section{DATA AND COMPUTER PROGRAM AVAILABILITY}

The resource link for code and data from this research is accessible on the following internet address https:/github.com/wianoski/geolocation-using-rssi-with-Trilateration-and-kalman-filter.

\section{ACKNOWLEDGMENT}

Thank you to the Internet of Things Studio, Telkom University which has become a place for researchers to develop this journal research. Hopefully, this research can make a major contribution to the advancement of technology in Indonesia

\section{REFERENCES}

[1] U. Raza, P. Kulkarni, and M. Sooriyabandara, "Low Power Wide Area Networks: An Overview," IEEE Commun. Surv. Tutorials, 2017, doi: 10.1109/COMST.2017.2652320.

[2] D. Croce, M. Gucciardo, S. Mangione, G. Santaromita, and I. Tinnirello, "Impact of LoRa Imperfect Orthogonality: Analysis of Link-Level Performance," IEEE Commun. Lett., 2018, doi: 10.1109/LCOMM.2018.2797057.

[3] I. W. K. Bima, V. Suryani, and A. A. Wardana, "A Performance Analysis of General Packet Radio Service (GPRS) and Narrowband Internet of Things (NB-IoT) in Indonesia," Kinet. Game Technol. Inf. Syst. Comput. Network, Comput. Electron. Control, 2020, doi: 10.22219/kinetik.v5i1.947.

[4] G. Li, E. Geng, Z. Ye, Y. Xu, J. Lin, and Y. Pang, "Indoor positioning algorithm based on the improved rssi distance model," Sensors (Switzerland), 2018, doi: 10.3390/s18092820.

[5] R. Oliveira, L. Guardalben, and S. Sargento, "Long range communications in urban and rural environments," 2017, doi: 10.1109/ISCC.2017.8024627.

[6] N. A. Azmi, S. Samsul, Y. Yamada, M. F. Mohd Yakub, M. I. Mohd Ismail, and R. A. Dziyauddin, “A Survey of Localization using RSSI and TDoA Techniques in Wireless Sensor Network: System Architecture," 2018, doi: 10.1109/TAFGEN.2018.8580464.

[7] H. C. Lee and K. H. Ke, "Monitoring of Large-Area IoT Sensors Using a LoRa Wireless Mesh Network System: Design and Evaluation,” IEEE Trans. Instrum. Meas., 2018, doi: 10.1109/TIM.2018.2814082.

[8] M. Turmudzi, A. Rakhmatsyah, and A. A. Wardana, “Analysis of Spreading Factor Variations on LoRa 
in Rural Areas,” 2019, doi: 10.1109/ICISS48059.2019.8969846.

[9] L. Yi, L. Tao, and S. Jun, "RSSI localization method for mine underground based on RSSI hybrid filtering algorithm,” 2017, doi: 10.1109/ICCSN.2017.8230129.

[10] L. J. Van Uffelen, B. M. Howe, E. M. Nosal, G. S. Carter, P. F. Worcester, and M. A. Dzieciuch, "Localization and subsurface position error estimation of gliders using broadband acoustic signals at long range,” IEEE J. Ocean. Eng., 2016, doi: 10.1109/JOE.2015.2479016.

[11] M. E. Rusli, M. Ali, N. Jamil, and M. M. Din, “An Improved Indoor Positioning Algorithm Based on RSSI-Trilateration Technique for Internet of Things (IOT),” 2016, doi: 10.1109/ICCCE.2016.28.

[12] K. H. Lam, C. C. Cheung, and W. C. Lee, "LoRa-based localization systems for noisy outdoor environment," 2017, doi: 10.1109/WiMOB.2017.8115843.

[13] G. P. Gerdan and R. E. Deakin, "Transforming cartesian coordinates X,Y,Z to geographical coordinates $\varphi, \lambda$, h,” Aust. Surv., 1999, doi: 10.1080/00050326.1999.10441904.

[14] B. C. Fargas and M. N. Petersen, "GPS-free geolocation using LoRa in low-power WANs,” 2017, doi: 10.1109/GIOTS.2017.8016251.

[15] G. A. Naik, M. P. Khedekar, M. Krishnamoorthy, S. D. Patil, and R. N. Deshmukh, "Comparison of RSSI techniques in wireless indoor geolocation," 2012, doi: 10.1109/NCCCS.2012.6413008.

[16] A. Awad, T. Frunzke, and F. Dressler, "Adaptive distance estimation and localization in WSN using RSSI measures,” 2007, doi: 10.1109/DSD.2007.4341511.

[17] É. Morin, M. Maman, R. Guizzetti, and A. Duda, "Comparison of the Device Lifetime in Wireless Networks for the Internet of Things," IEEE Access, 2017, doi: 10.1109/ACCESS.2017.2688279.

[18] K. H. Lam, C. C. Cheung, and W. C. Lee, "New RSSI-Based LoRa Localization Algorithms for Very Noisy Outdoor Environment," 2018, doi: 10.1109/COMPSAC.2018.10340.

[19] M. Md Din, N. Jamil, and N. F. Nik Ahmad Aziz, "Adopting Centroid and Amended Trilateration for better accuracy of range-based non-GPS localization,” 2018, doi: 10.1109/ICCOINS.2018.8510605.

[20] A. Mackey and P. Spachos, "LoRa-based Localization System for Emergency Services in GPS-less Environments," 2019, doi: 10.1109/INFCOMW.2019.8845189.

[21] E. Goldoni, L. Prando, A. Vizziello, P. Savazzi, and P. Gamba, "Experimental data set analysis of RSSIbased indoor and outdoor localization in LoRa networks," Internet Technol. Lett., 2019, doi: 10.1002/it12.75.

[22] W. Bulten, A. C. Van Rossum, and W. F. G. Haselager, "Human SLAM, indoor localisation of devices and users," 2016, doi: 10.1109/IoTDI.2015.19.

[23] J. Du, J. F. Diouris, and Y. Wang, “A RSSI-based parameter tracking strategy for constrained position localization," EURASIP J. Adv. Signal Process., 2017, doi: 10.1186/s13634-017-0512-x.

[24] K. C. Lee, A. Oka, E. Pollakis, and L. Lampe, “A comparison between unscented Kalman filtering and particle filtering for RSSI-based tracking,” 2010, doi: 10.1109/WPNC.2010.5650817. 